\title{
Experimental study of the turbulent flow around a single wall-mounted prism obstacle placed in a cross-flow and an impinging jet
}

\author{
Y. Masip, A. Rivas, A. Bengoechea, R. Antón, \\ G. S. Larraona \& J. C. Ramos \\ Thermal and Fluids Engineering Division, Mechanical Department, \\ TECNUN-University of Navarra, San Sebastián, Spain
}

\begin{abstract}
This paper describes the experimental part of an electronics cooling research project. To undertake the experiments a test rig has been designed and assembled which represents a channel made by two parallel printed circuit boards (PCBs). Different arrangements of heated prismatic bodies can be mounted on the walls of the channel simulating electronic components. These bodies are cooled combining a channel cross-flow and impinging jets issuing from the walls. The working fluid is air and the maximum Reynolds number (calculated respect to the channel height) is 13740 . The test rig has been designed to take flow measurements using different experimental techniques such as hot wire anemometry (HWA), particle image velocimetry (PIV) and infrared imaging (IR). Experimental data on fluid flow features around electronic components will be used to develop and validate turbulence models that will be implemented in Computational Fluid Dynamics simulations.

The presented results are two preliminary experimental studies to analyse the influence of the component height $(h)$ and the Reynolds number on the flow structure around the component. The first study was made with the component in a cross flow without impinging jet and in the second study an impinging jet was added to the cross flow. The Reynolds numbers employed are close to those used in applications within the electronics industry. The measurement mean $(U, V)$, rms velocity $\left(u_{r m s}, v_{r m s}\right)$ profiles in three main regions of the flow, namely, the wake, the upper and the side region have been obtained using the hot wire anemometry. From theses measurements the lateral flow separation, reattachment points and recirculation have been studied.

Keywords: electronic cooling, cross flow, impinging jet, wall-mounted prism.
\end{abstract}




\section{Nomenclature}

\begin{tabular}{|c|c|c|c|}
\hline$H, W$ & $\begin{array}{l}\text { Channel height and width } \\
\text { (mm) }\end{array}$ & $U_{\max }$ & $\begin{array}{c}\text { Maximum Velocity at centerline } \\
\text { of the channel }(\mathrm{m} / \mathrm{s})\end{array}$ \\
\hline$L, h$ & $\begin{array}{l}\text { Obstacle width and height } \\
(\mathrm{mm})\end{array}$ & $U_{j}$ & Impinging Jet Velocity $(\mathrm{m} / \mathrm{s})$ \\
\hline$h / L$ & Obstacle aspect ratio & $x, y, z$ & $\begin{array}{l}\text { Streamwise, Vertical and } \\
\text { Spanwise coordinates (mm) }\end{array}$ \\
\hline$R e_{H}$ & $\begin{array}{l}\text { Channel Reynolds number } \\
\qquad\left(=U_{m} H / v\right)\end{array}$ & $v$ & Fluid kinematic viscosity $\left(\mathrm{m}^{2} / \mathrm{s}\right)$ \\
\hline$R e_{i}$ & $\begin{array}{l}\text { Impinging jet Reynolds } \\
\text { number }\left(=U_{j} D / v\right)\end{array}$ & & \\
\hline$U_{m}$ & $\begin{array}{l}\text { Channel Bulk velocity } \\
\qquad(\mathrm{m} / \mathrm{s})\end{array}$ & & \\
\hline
\end{tabular}

\section{Introduction}

Thermal management of electronic systems is one of the bottlenecks in today's electronics industry. The increasing clock speeds and miniaturization implies a huge increase of the heat dissipation per unit area in electronic systems. It is a real challenge to cool electronic components in an efficient way, since the heat transfer in electronic components depends strongly on characteristics of the flow field around them. That is the reason why the flow and heat transfer around small-scale three-dimensional blocks, which simulated electronic components, has aroused great interest.

Flows over isolated, wall-mounted cubes have been subject to several past investigations. Castro and Robins [1] measured the flow velocity around a single cube mounted on a flat plate with different oncoming boundary layer flows. It was shown that the extent of the wake recirculation and the size of the vortex on top of the obstacle were strongly dependent on the boundary layer thickness of the oncoming flow. Ogawa et al. [2] studied the flow and mass diffusion around a cube considering the concentration and flow patterns on and around the cube. The concentration patterns were shown to be strongly dependent upon the flow patterns, and in particular, on the existence of reverse flow at the source location.

The flow around a single wall-mounted cube in a fully developed turbulent channel flow was investigated experimentally by Hussein and Martinuzzi [3], Larousse et al. [4], Martinuzzi [5] and Martinuzzi and Tropea [6]. The Reynolds number was $R e_{h}=40000$, based on the cube height $(h=25 \mathrm{~mm})$ and the bulk velocity, and the channel height-to-cube ratio was 2 . The major flow features were well described, namely the horseshoe vortex induced at the leading face of the cube, the arc-shaped vortex in the wake, the flow recirculations at the top and the side faces. Further, the studies included a detailed description of the Reynolds stresses and other higher order moments. It was found that the horseshoe vortex region was unstable but non-periodic which caused bimodal 
velocity distributions. Moreover, vortex shedding was detected in the wake of the cubical obstacle. Meinders [7] and Meinders et al. [8-10] reported on the flow field characteristics (e.g.: flow separation and reattachment at the top and side faces of the cube) of a single wall-mounted cube $(h=15 \mathrm{~mm})$ in a developing turbulent channel flow for a Reynolds number range of $2750<R e_{h}<4970$.

The literature review of Schofield and Logan [11] showed that a consensus exists for the general flow features around a single wall-mounted prism. The features include the horseshoe vortex, the wake vortex and the top and side vortices, which are indicated in Figure 1. These flow features are not only observed for a single prism in turbulent channel flows, but are also representative for configurations of multiple prisms. The flow field in front of the obstacle is characterized by the horseshoe vortex, indicated with $\mathbf{A}$, which originates at the base of the windward stagnation region and extends downstream along the channel floor past the sides of the prism. The separation of the top shear layer resulted in a bound recirculation ("top vortex", indicated with B). The side vortex is indicated with $\mathbf{C}$. An arc-shaped vortex was typically found in the wake of the obstacle ("wake vortex"). This wake vortex, indicated with D, was confined by the solid walls and the fluid flowing along the side and the top faces.

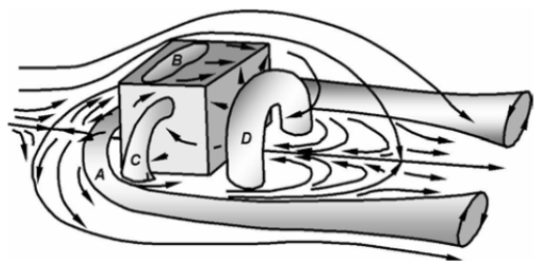

Figure 1: Schematic representation of the flow features a surface-mounted obstacle by Martinuzzi and Tropea (From [6] with permission).

In cooling of electronic components, forced channel flow between PCBs is frequently used as a cooling technique [7]. Using forced channel flow excessive flow rates will be required to manage the whole thermal load of the electronic system. One possible alternative to face this problem is using an impinging jet over the most dissipating component combined with a low-velocity channel flow (see Rundström and Moshfegh [12]). Impinging jets are used for many industrial applications where high heat and mass transfer rates are required. In addition to their industrial applications, turbulent impinging jets have great scientific interest due to the particular characteristics of this flow. Extensive experimental and numerical research has been carried out to predict the flow and heat transfer in the stagnation region of an impinging jet.

This paper presents an experimental study about the influence of the obstacle height and Reynolds number on the flow field structure. The component used is located in a cross flow for the first case and in the second case the component is placed in a cross flow adding an impinging jet. The flow features determined for the first case were the reattachment point on the top and downstream of the trailing face of the component and the lateral separation. Moreover, a 
comparison with the results found in the literature was performed. In the second case two principal regions, the upper zone and the wake were studied.

\section{Experimental setup}

\subsection{Test section description}

The experiments were performed in an open circuit wind tunnel specifically designed for electronics applications. This wind tunnel was built following the criteria found in Mehta and Bradshaw [13], Westphal [14], Barlow et al. [15]. As can been seen in Figure 2, the wind tunnel consisted of different assembled pieces, namely input cone, honeycomb, screens, contraction, test section, diffuser, filters, laminar flow element and centrifugal fan.
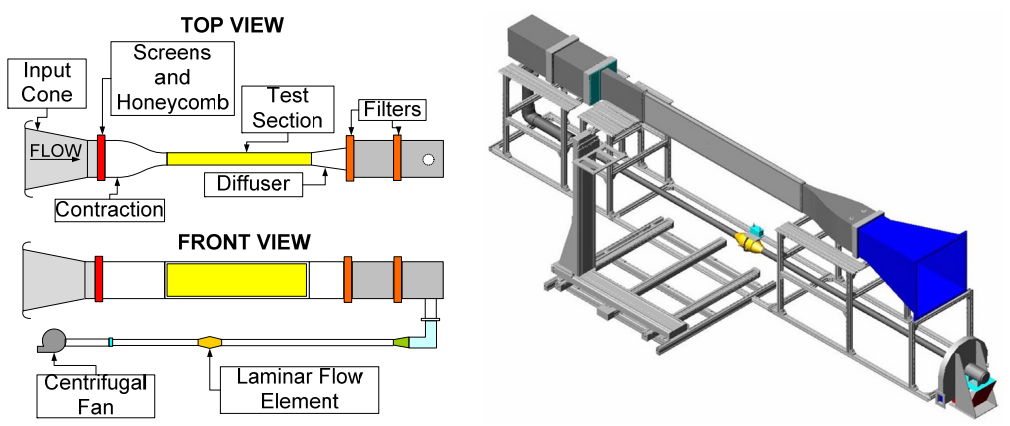

Figure 2: $\quad$ View of the wind tunnel.

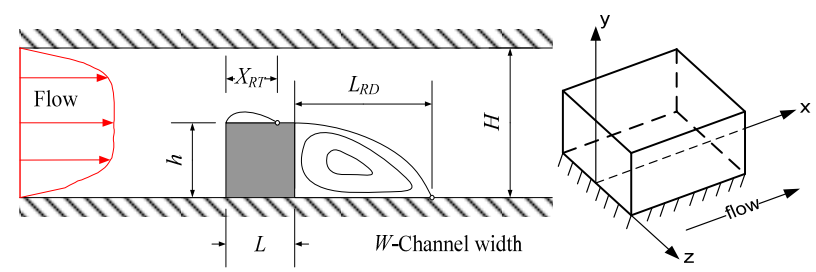

Figure 3: Sketch of the side view of the test-section with a single wallmounted obstacle and local coordinate system of the obstacle.

The honeycomb and fine screens were used to eliminate the large turbulence scales and to straighten the flow. The channel (test section) has internal dimensions of $2000 \times 30 \times 300 \mathrm{~mm}^{3}$. A sketch of the test section with the coordinate system employed is given in Figure 3 . The $x$-axis and $y$-axis refer to the streamwise and the normal direction respectively. The $z$-axis points out of the image, referring to the spanwise direction. The diffuser is used for the transition between a section of high velocity and one of low velocity. The filters are used to eliminate the strange particles conveyed by the air and to avoid damage of the 

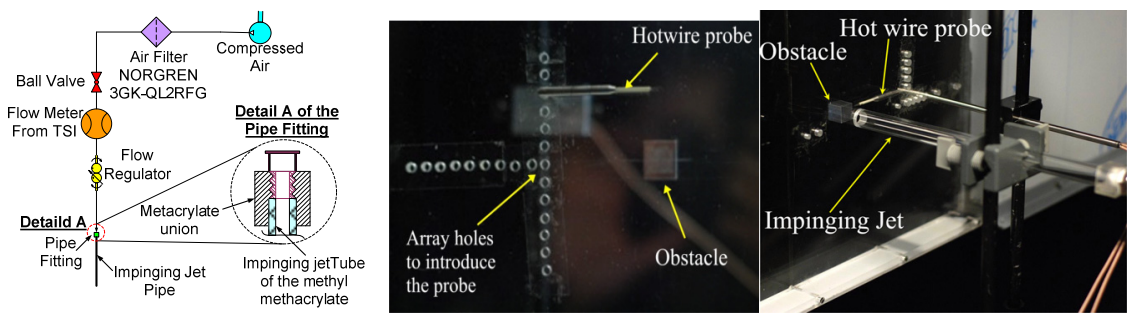

Figure 4: Sketch of the control system of the flow for the impinging Figure 5: Photograph of the test-section with a single wall-mounted obstacle during the hot wire measurements. jet.

laminar flow element. The laminar flow element is used to measure the volumetric flow that circulating through wind tunnel. The centrifugal fan has a variable-frequency driver so that different flow rate can be achieved.

The test section has been designed and assembled to represent a channel made by two parallel PCBs. Different arrangements of heated prismatic bodies can be mounted on the walls of the channel simulating electronic components. The walls are made of a transparent material (methyl methacrylate) to have optical access to the test section. This setup provides the possibility to assemble and disassemble the walls with little effort. The front wall has an array of holes to introduce the support of the hot wire probe. Also one plate can be changed in order to use the impinging jet. The impinging jet enters to the test section through a rigid tube sufficiently long to obtain fully developed flow. The flow rate of the impinging jet is controlled by means of a flow meter, valve and flow regulator to maintain the required working conditions, see Figure 4. A photograph of the test section with both setups is shown in Figure 5.

A computer application has been developed in Labview 8 to control and monitor the working flow rate through both the channel and the impinging jet.

\subsection{Measurement equipment and uncertainty analysis}

Mean velocity and second order statistic were measured with a HWA. This equipment includes a Streamline Calibrator, Streamline Module (type 9010), A/D board to convert analogical signal into digital and Probe (X-probe type 55P61 two-dimensional velocity component). The probe used has the possibility of measuring two-dimensional flows, where the velocity vector stays within $\pm 45^{\circ}$ with respect to the probe axis (see Jorgensen [16]). Due to the rotational symmetry of the sensing element (Bruun [17]), the cylindrical hot-wire probe cannot detect negative velocities. Therefore, the location of small recirculation regions will be estimated from the Reynolds stresses in the wake downstream of the trailing face and over the top face [1].

The uncertainty value of the calibration equipment has been provided by the manufacturer and is $1 \%$. The data acquisition is related to $\mathrm{A} / \mathrm{D}$ board resolution 
which has an uncertainty of $0.13 \%$ calculated following Jorgensen [16]. The uncertainties related to experimental conditions depend on the ambient conditions and the probe positioning. The ambient conditions are measured automatically by the equipment. The positioning uncertainty $(\Delta \theta)$ relates to the alignment of the probe in the experimental setup after calibration. This value is estimated from measurements in the centerline of the channel being approximately $\Delta \theta \approx 2.5^{\circ}$. Finally, the total uncertainty is approximately $2.7 \%$.

The volumetric air flow rate was measured by a laminar flow element with a total accuracy, including the pressure transducer, of $\pm 0.87 \%$ of the reading. The humidity, atmospheric pressure and ambient temperature were recorded in order to make corrections.

\subsection{Channel and impinging jet flow conditions}

The features of the oncoming flow in the channel are found upstream of the obstacle at a far plane where the flow is unperturbed by the latter. The measurements are made for Reynolds numbers close to those used in electronics industry applications, namely $R e_{H}=3410,4560,5752$ and 8880 with respect to channel height and bulk velocity. The mean velocity and Reynolds stress profiles in the channel are measured $120 \mathrm{~mm}$ upstream of the front face of the obstacle. A typical profile at the centerline of the channel is given in Figure 6 . The analysis of the velocity and Reynolds stresses shows that the channel flow is under development. In the case of the impinging jet it is impossible to measure the velocity profile at the jet inlet with the hot wire because the probe introduced in the tube alters the flow stream.
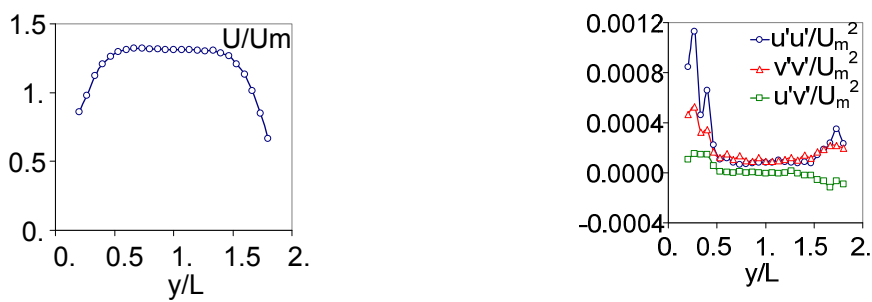

Figure 6: $\quad$ Profile of the dimensionless streamwise velocity and Reynolds stresses in the normal direction at $x / L=-8$ at $z / L=0$ for $R e_{H}=8880$.

\section{Test procedure}

Hot wire measurements were performed in different planes $x / L, y / L$ and $z / L$ (see Table 1) for the wake, top and side regions around of the obstacle respectively.

The planes are chosen in such a way the measurements can be taken as close as possible to the obstacle and it was able to locate the hot wire probe. The flow and turbulence fields have been built from measurements obtained in a large number of points. The employed spatial resolution in the $y$-axis direction has been $1 \mathrm{~mm}$. The combination of the resolution, the measuring planes and the Reynolds numbers produces 6360 points where mean velocity, Reynolds stresses 
and higher order moments in $x$ and $y$ directions were measured. These values are the average of 5000 samples taken at a frequency of $1 \mathrm{kHz}$. The impinging jet measurements are taken in the central plane $z / L=0$. It is important to highlight that due to the hot wire probe diameter $(3 \mathrm{~mm})$ it is impossible to measure in the near-wall region. It is one of the main limitations in employing this intrusive technique.

Table 1: $\quad$ Measurement plane positions.

\begin{tabular}{c|c|c}
\hline Wake $(z / L)=0$ & Upper $(z / L)=0$ & Side $(x / L)=0.5$ \\
$x / L$ & $x / L$ & $z / L$ \\
\hline \hline 1.1 & 0 & 0.9 \\
1.5 & 0.25 & 1 \\
2 & 0.5 & 1.5 \\
3 & 0.75 & 2 \\
5 & 1 & 2.5 \\
& & 3 \\
\hline
\end{tabular}

\section{Results and discussion}

\subsection{Case 1}

The first experimental study is about the flow field around a prismatic obstacle mounted on the wall of the flow channel. In this study all components have constant width and length $L=H / 2=15 \mathrm{~mm}$ but different height $(h)$ namely 5,10 , $15 \mathrm{~mm}$. The aim of this study is to determine the influence of the height of the obstacle and the Reynolds number on the flow structure around the component. The flow separation on the sides of the obstacle and the reattachment point downstream from the trailing face zones have been studied in this case.

\subsubsection{Wake of the obstacle}

The mean flow pattern in the wake recirculation region is dominated by an arcshaped vortex as shown in Figure 1. This vortex was produced by separation of the shear layer at the top trailing edge and reattached downstream of the trailing face. In this situation it is possible to obtain the reattachment point and hence the recirculation length to characterize the wake. The mean velocity $\left(U / U_{m}\right)$ and Reynolds normal stress $\left(u_{r m s}{ }^{2} / U_{m}{ }^{2}\right)$ profiles are given several $y / L$ locations in the symmetry plane $z / L=0$ in Figures 7 and 8 respectively.

The reattachment length depends on the interaction between the shear layer released at the trailing edge of the component and the main stream. The bulk flow forces the shear layer to reattach at the floor of the channel downstream of the back face. Normally, the reattachment point is found where the negative velocities disappear in the wake. The reattachment point can be estimated in the region that the Reynolds stresses present maximum values (e.g. Figure 8) and the velocity profile stabilizes [1]. This parameter is important because the recirculation in the wake is located between the reattachment point and the 

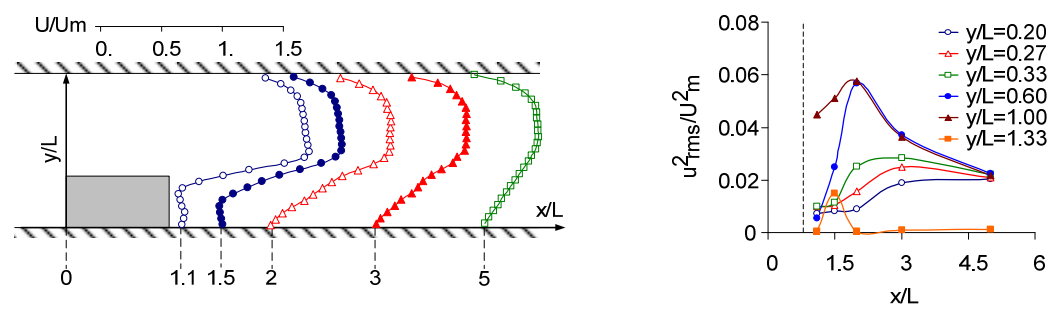

Figure 7: $\quad U / U_{m}$ at $z / L=0, h=10 \mathrm{~mm}, \quad$ Figure 8: $\quad u_{r m s}{ }^{2} / U_{m}{ }^{2}$ at $z / L=0$, $R e_{H}=8880$. $h=10 \mathrm{~mm}, \operatorname{Re}_{H}=8880$.
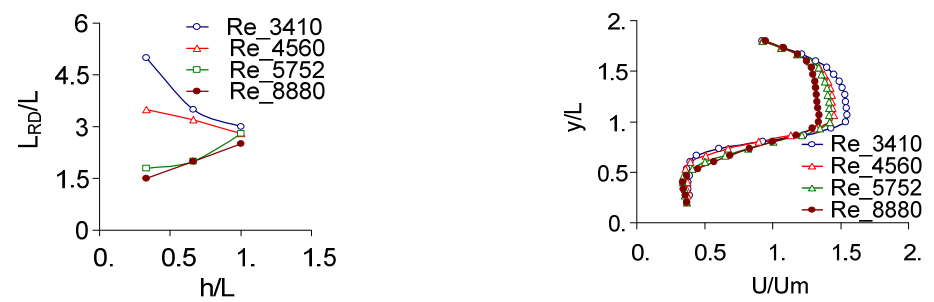

Figure 9: Length of reattachment as function of $h / L$ and $R e_{H}$.

Figure 10: $U / U_{m}$ for different Reynolds numbers for $h=10 \mathrm{~mm}$ at $x / L=1.5$.

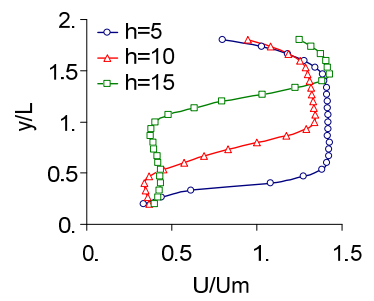

Figure 11: $\quad U / U_{m}$ for different component heights at $x / L=1.5$ and $R e_{H}=8880$.

trailing face and it is limited in the vertical plane by the shear layer. The region of maximum values depends of the obstacle height and occurs approximately at $y / h=0.9-1.2$. The normalized reattachment length $\left(L_{R D} / L\right)$ is plotted as a function of the aspect ratio $(h / L)$ and Reynolds number in Figure 9, where it can be seen that as the value of $h / L$ increases the reattachment length shows almost no change with the Reynolds number. In the case of components with low height, the Reynolds number has a major influence in the reattachment length (Figures 10 and 11 show this fact).

\subsubsection{Top face of the obstacle}

The top face is characterized by a bound vortex in the vicinity of the leading edge. Figure 1 shows this vortex type. A shear layer appears due to the flow 
detachment at the leading edge of the component. The flow reattaches almost at the trailing edge and it is possible to identify the point of reattachment because in this region are found the maximum values of the Reynolds stress, given in Figure 13. In the case of height $5 \mathrm{~mm}$ this point is almost undetectable, although it occurs at the same location. The central zone of the upper recirculation is observed over the top face at $x / L=0.5$ plane where the velocities are minimum for the three obstacles used, see Figure 12. The normal Reynolds Stress profiles show maxima approximately at $y / h=1-1.2$, which coincides with areas of local production of turbulence for the three heights caused by the reattach of the flow. The influence of Reynolds number and component height on the velocity profile is shown in Figures 14 and 15 respectively. The Reynolds number does not have a significant influence on the flow near the top of the component. However, the increase of height causes a variation in the flow structure.

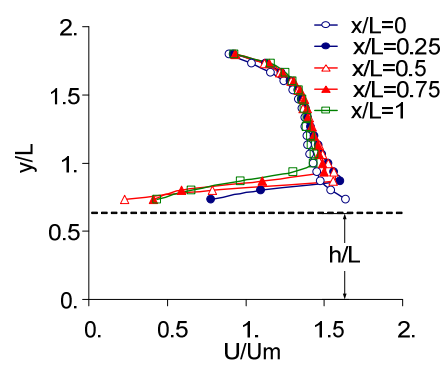

Figure 12: $U / U_{m}$ in the top of the obstacle at $z / L=0$, $h=10 \mathrm{~mm}, R e=5752$.

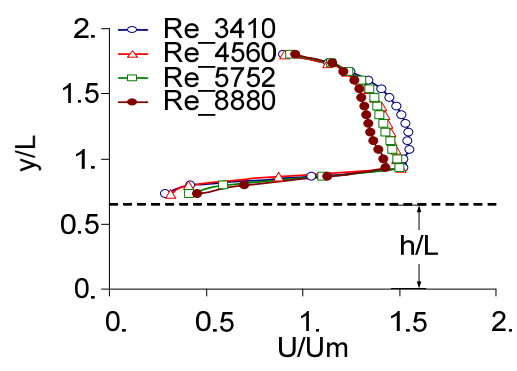

Figure 14: $U / U_{m}$ for different Reynolds numbers at $x / L=0.75$ and $h=10 \mathrm{~mm}$.

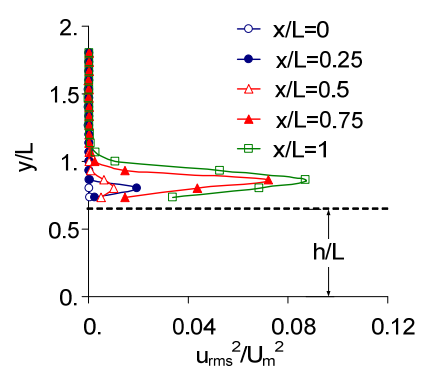

Figure 13: $\quad u_{r m s}{ }^{2} / U_{m}{ }^{2}$ in the top of the obstacle at $z / L=0$, $h=10 \mathrm{~mm}, R e=5752$.

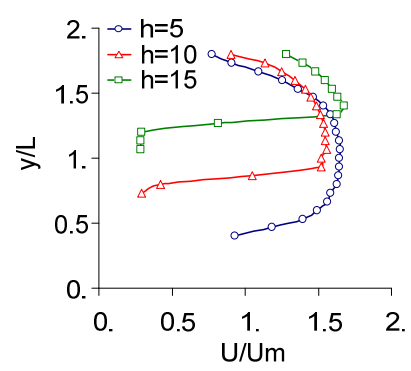

Figure 15: $U / U_{m}$ for different component heights at $x / L=0.75$ and $R e=3410$. 

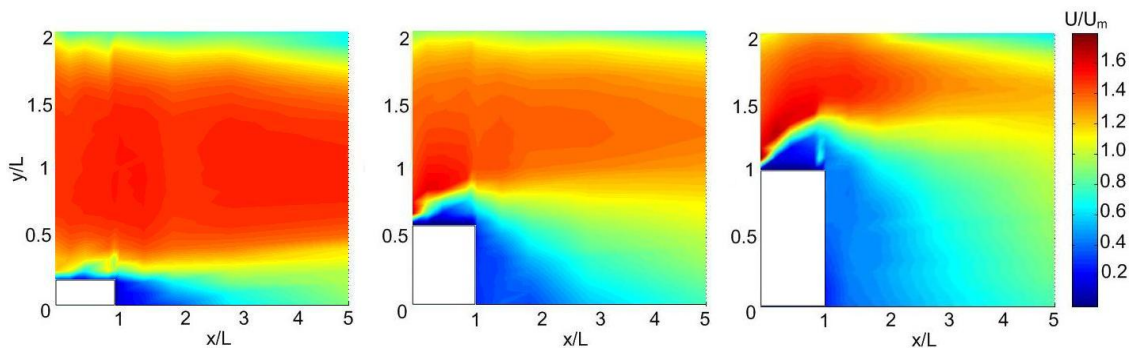

Figure 16: Contours of the $U / U_{m}$ for the component $h=5,10$ and $15 \mathrm{~mm}$ respectively at $z / L=0$ and $R e=5752$.

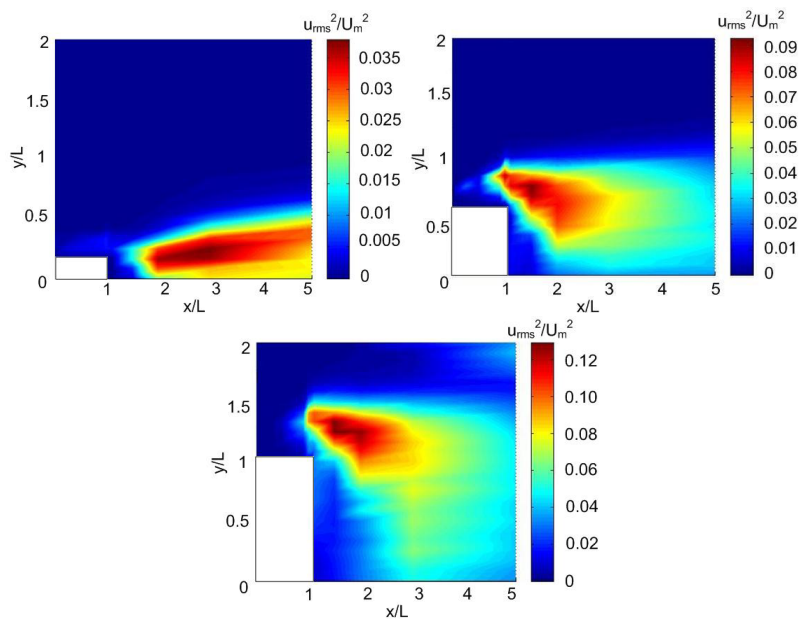

Figure 17: Contours of the $u_{r m s}{ }^{2} / U_{m}{ }^{2}$ for the component $h=5,10$ and $15 \mathrm{~mm}$ respectively at $z / L=0$ and $R e=5752$.

Figures 16 and 17 depict the velocity and normal Reynolds stress contours for the three component heights built from measurements. These pictures show the shear layer shedding at the leading edge and the approximate reattachment point in the wake.

\subsubsection{Side of the obstacle}

The flow at both sides of the obstacle is dominated by a side vortex close to the leading edge, confined by the main stream and the obstacle. An important parameter is the lateral separation $(Z s)$ which allows establishing the zone perturbed by the horseshoe vortex. This vortex appears due to the impact of the flow with the front face of the obstacle. The velocity and Reynolds stress profiles for the lateral region are given in Figures 18 and 19 respectively. In Figure 20 the influence of the component height on the velocity profile is shown, and this influence is significant because a larger height increases both the blockage and the separation of the flow, causing decrease in the velocity on the sides of the 


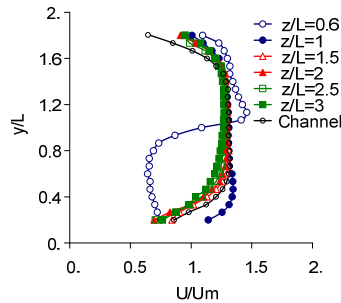

Figure 18: $U / U_{m}$ in the side at plane $x / L=0.5, h=15 \mathrm{~mm}$ and $R e=8880$.

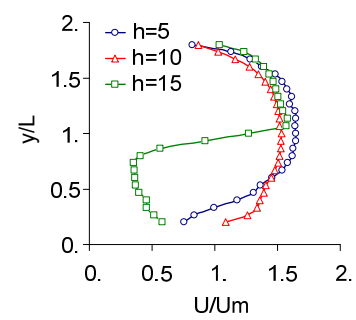

Figure 20: $U / U_{m}$ for different component heights at $x / L=0.5, \quad \mathrm{z} / L=0.6$ and $R e=3410$.

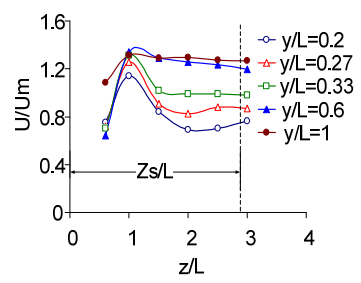

Figure 22: $U / U_{m}$ at the lateral face at $x / L=0.5, \quad h=15 \mathrm{~mm}$ and $R e=8880$.

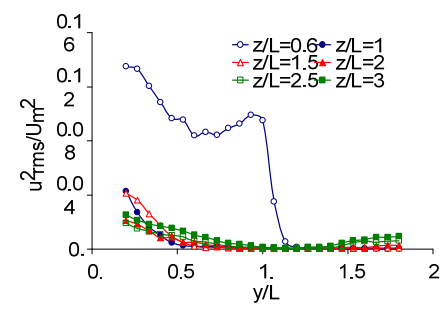

Figure 19: Of $u_{r m s}{ }^{2} / U_{m}{ }^{2}$ in the side at $x / L=0.5, h=15 \mathrm{~mm}$ and $\mathrm{Re}=8880$.

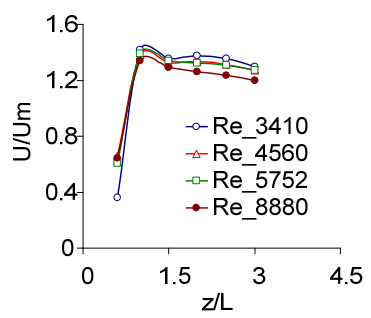

Figure 21: $U / U_{m}$ for different Reynolds numbers for $\mathrm{h}=15 \mathrm{~mm} \quad$ at $\quad x / L=0.5$, $y / L=0.6$.

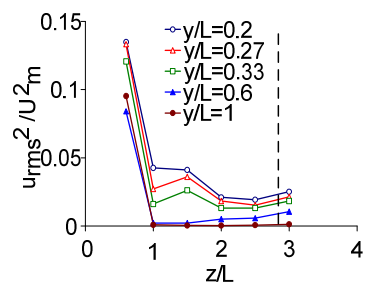

Figure 23: Reynolds stresses at $x / L=0.5, \quad h=15 \quad$ and $R e=8880$.

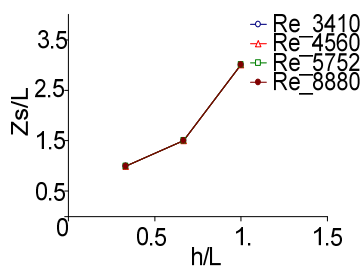

Figure 24: Length of the spanwise separation as function of $h / L$. 
component. The Reynolds number almost shows no effect on the velocity profile in the side, see Figure 21.

The lateral separation is found from measurements of the mean velocities and normal Reynolds stresses given in Figures 22 and 23 respectively for six spanwise planes. The Reynolds stress profiles show their maximum values in the vicinity of the obstacle wall, at approximately $y / h=0.9$ for the three obstacle heights. The estimation of this separation is found from the point at which the velocity profile is uniform and the Reynolds stress profile decreases and almost remains at the same value. In Figures 22 and 23 the profiles keep a stable value from the broken line and the distance to this point coincides with the length of the lateral separation.

In Figure 24 the normalized spanwise separation length $(Z s / L)$ is plotted as a function of the aspect ratio and the Reynolds numbers. It can be seen that the lateral separation length increases with the increasing obstacle height. The curves for all Reynolds numbers are almost superimposed indicating that the latter does not have a significant influence on the lateral separation.

\subsubsection{Comparison with the literature}

The single component in the cross flow case discussed in this study differed in several aspects from the case of Martinuzzi and Tropea [6]. The component in the present study is placed in a developing turbulent channel using different Reynolds numbers, while Martinuzzi and Tropea considered a fully developed channel flow for a Reynolds number which is almost one order of magnitude higher $\operatorname{Re}_{H}=80000$. However, the channel-height-to-cube-size ratio was equal in both studies $(H / h=2)$.

The present study has found a reattachment flow on the top face of the component that was absent in the fully developed case [7]. Further, the flow reattachment at the channel floor downstream from the component occurs at a larger distance away from the trailing face than in the fully developed channel flow.

Profiles of the mean velocity $U / U_{\max }$ are compared in Figure 25 with the results from Martinuzzi and Tropea. The coordinate is scaled with the component width. The differences in the proximity of the component face are caused by use of the hot wire. The agreement in the other regions is good.

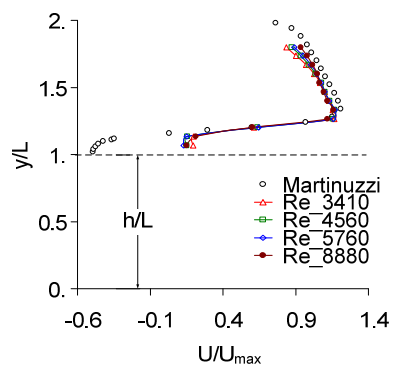

Figure 25: Comparison between the present study and the literature result: traverses of $U / U_{\max }$ in the $\mathrm{z} / L=0$ and $x / L=0.5$. 


\subsection{Case 2}

The second experimental case is the study of the flow around a wall-mounted component in a cross-flow adding an impinging jet. This configuration represents a simplified case of an electronics cooling device. The sketch of the flow under consideration is shown in Figure 26. It consists of two fluid streams: the first is a channel flow over a single wall-mounted cube, and the second is a round jet impinging perpendicular and centred on the cube. The Reynolds number of the channel flow is $R e_{H}=3410$, based on its bulk velocity $U_{m}=1.66$ $\mathrm{m} / \mathrm{s}$ and the channel height $2 h=30 \mathrm{~mm}$. The Reynolds number of the impinging jet is $R e_{j}=3936$, based on its bulk velocity $U_{j}=4.79 \mathrm{~m} / \mathrm{s}$. These Reynolds numbers and velocity ratios correspond roughly to real electronics cooling systems. The obstacles employed have equal width, length and height $(L=h=15$ $\mathrm{mm})$.

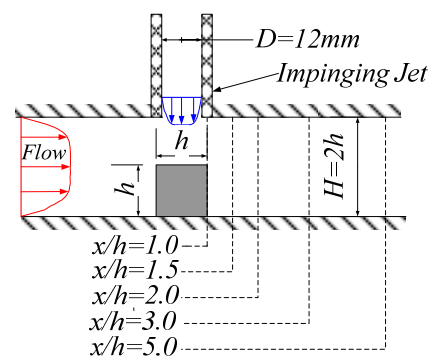

Figure 26: Sketch of the experimental setup and measurements $x y$-planes.
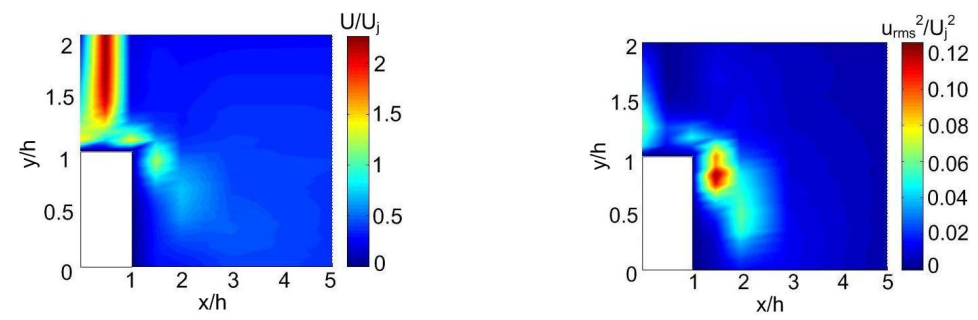

$\begin{array}{ll}\text { Figure 27: } \begin{array}{l}\text { Contours of the } U / U_{j}^{2} \text { in } \\ \text { the } x y \text {-plane, } z / L=0 .\end{array} & \text { Figure 28: } \begin{array}{l}\text { Contours of the } u_{r m s}{ }^{2} / U_{j}^{2} \\ \text { in the } x y \text {-plane, } z / L=0 .\end{array}\end{array}$

The velocity field is illustrated by contour plots of the velocity and Reynolds stress in the $x y$-plane at the centre of the channel (see Figures 27 and 28) obtained from measurements. The flow field shows a complex behaviour and there are several flow-related phenomena that can affect the cooling performance, for example the position of the stagnation point on top of the component and the separation of the flow at the trailing edge of the component.

The cross-flow affects to the impinging jet and the separation from the top of the cube. The impinging jet creates a downstream flow with a zone of the 
Reynolds stress that is high (approximately at $y / h=0.9$ ) due to the shear layer and vortex shedding in the impingement process. The recirculation is almost not detected in the region behind the rear side of the cube due the low values employed for the Reynolds number. The stagnation region over the top face is detected where the velocity values and the Reynolds stresses are almost insignificant.

The cross-flow affects to the impinging jet and the separation from the top of the cube. Downstream the impinging jet creates a zone of high Reynolds stress approximately at $y / h=0.9$ due to the shear layer and vortex shedding in the impingement process. Due to the low value of the Reynolds number, the recirculation in the region behind the trailing face of the obstacle is difficult to detect. The stagnation region over the top face is detected where the velocity values and the Reynolds stresses are very low.

Figure 29 shows the normalized $x$-velocity component $\left(U / U_{j}\right)$ as a function of the vertical distance $(y / h)$. Each diagram represents different locations in the $x$ direction at $z / h=0$. The first diagram corresponds to the zone where the flow is accelerated by the impact of the jet on the top of the component. This result is in agreement with the experimental study of Tummers et al. [18]. The region where the flow is accelerated have almost identical values due to the interaction of the shear layer (see second diagram in Figure 29).

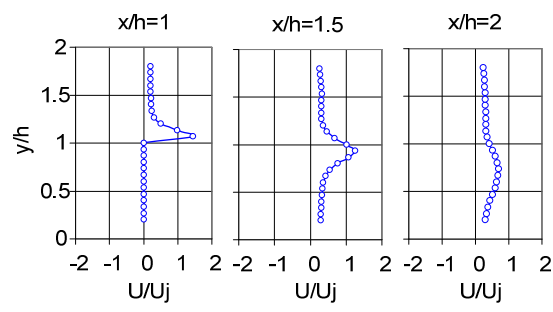

Figure 29: $x$-velocity components $\left(U / U_{j}\right)$ in the $x y$-plane, $z / h=0$.
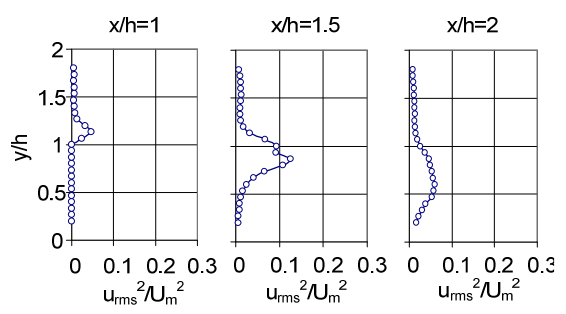

Figure 30: Reynolds stress $\left(u_{r m s}{ }^{2} / U_{j}^{2}\right)$ in the $x y$-plane, $z / h=0$.

Figure 30 shows the normalized Reynolds stress $\left(u_{r m s}{ }^{2} / U_{j}^{2}\right)$ as a function of the vertical distance $(y / h)$ at the centreline. The Reynolds stresses at $x / h=1$ show a maximum value over the top face. This zone corresponds with the interaction between the impinging jet flow and the top face of the obstacle (see the first diagram in Figure 30). The maximum value of Reynolds stresses is found approximately at $x / h=1.5$ and $y / h=0.9$ (see the second diagram in Figure 30) which indicates where there exists a maximum production of $u_{r m s}{ }^{2} / U_{j}^{2}$. This occurs as a result of the interaction between the shear layer released by the impinging jet over the top face and the cross flow in the wake downstream of the trailing face. 


\section{Conclusions}

The flow around an obstacle with different heights $(h=5,10$ and $15 \mathrm{~mm})$ was studied experimentally employing several Reynolds numbers. This study has shown that for high height the reattachment length downstream of the trailing face remains almost unchanged for the Reynolds numbers. However, for small height the Reynolds numbers has a high influence on the $L_{R D}$, in this case when the Reynolds numbers decrease the reattachment length increase. For the studied obstacle heights, points which have the maximum values of Reynolds stresses exit in the wake and top of the obstacle, their positions are a function of the obstacle height. In the case of the wake, these points are located at $y=1.2$ of the channel floor downstream of the trailing face. At the top face of the component it appears approximately $0.2 h$ above this face.

The lateral separation length increases with the obstacle height. The Reynolds number has no influence on the lateral separation. The normal Reynolds stresses show their maximum values close to the side faces, approximately $0.9 \mathrm{~h}$ above the channel floor where the highest turbulence production was found, due to the shear layer interaction.

In the case of impinging jet and cross flow combination the measurements indicate the presence of a stagnation point at the centre of the top face of the obstacle and regions of turbulence production downstream of the trailing face. The downstream region of the trailing face have high Reynolds stresses at approximately $x / h=1.5$ and $y / h=0.9$. This region corresponds to the maximum turbulence production due to the shear layer and vortex shedding. These observations agree with those found in other studies.

\section{Acknowledgements}

This research was funded by Ministerio de Ciencia e Innovacion, Spain Government through program CICYT D+I (DPI2008-05349). The support of Cátedra Fundación Antonio Aranzabal-Universidad de Navarra and Linköping University (Sweden) is also gratefully acknowledged.

\section{References}

[1] Castro, I.P., Robins, A.G. The flow around a surface-mounted cube in uniform and turbulent streams. J. Fluid Mech. 79 (2), pp.307-335, 1977.

[2] Ogawa, Y., Oikawa, S., Uehara, K. Field and wind tunnel study of the flow and diffusion around a model cube-II. Near field and cube surface flow and concentration patterns. Atmospheric Environment. 17 (6), pp. 1161-1171, 1983.

[3] Hussein, H.J., Martinuzzi, R.J. Energy balance for turbulent flow around a surface mounted cube placed in a channel. Phys. Fluids 8 (3), pp. 764-780, 1996.

[4] Larousse, A., Martinuzzi, R., Tropea, C. Flow around surface-mounted, three-dimensional obstacles. In: Eighth Symposium on Turbulent Shear Flows. TU-Munich/Germany, vol. 1, pp. 1441-1446, 1991. 
[5] Martinuzzi, R.J. Experimentelle Untersuchung der Umstroming wandgebundener, rechteckiger, prismatischer Hindernisse. Ph.D. Thesis, Erlangen, Germany.

[6] Martinuzzi, R.J., Tropea, C. The flow around surface mounted, prismatic obstacles placed in a fully developed channel flow. J. Fluids Eng. 115, pp. 85-92, 1993.

[7] Meinders, E.R. Experimental study of heat transfer in turbulent flows over wall-mounted cubes. Ph.D. Thesis, Faculty of Applied Sciences, Delft University of Technology, Delft, Netherlands, 1998.

[8] Meinders, E.R., van der Meer, T.H., Hanjalic, K., Lasance, C.J.M. Application of infrared thermography to the evaluation of local convective heat transfer on arrays of cubical protrusions. Int. J. Heat and Fluid Flow 18 (1), pp. 152-159, 1997.

[9] Meinders, E.R., van der Meer, T.H., Hanjalic, K. Local convective heat transfer from an array of wall-mounted cubes. Int. J. Heat and Mass Transfer 41 (2), pp. 335-346, 1998.

[10] Meinders, E.R., Martinuzzi, R., Hanjalic, K. Experimental study of the local convective heat transfer from a wall-mounted cube in turbulent channel flow. Int. J. Heat and Mass Transfer 45, pp. 465-482, 2002.

[11] Schorfield, W.H., Logan, E. Turbulent shear flow over surface mounted obstacles. J. of Fluids Engineering, pp. 113, 405, 1990.

[12] Rundström, D., Moshfegh, B. RSM and $v^{2}-f$ study on the flow behavior of an impinging jet in a cross-flow on a wall-mounted cube. Progress in Computational Fluids Dynamics, vol7, No.6. pp. 311-322, 2007.

[13] Mehta, R.D., Bradshaw, P. Design Rules for Small Low Speed Wind Tunnels. Aeronautical Journal, pp. 443-449, 1979.

[14] Westphal, R.V. Wind tunnel design. Proceedings, Thermal measurements in electronic cooling. (K. Azar, editor), Boca raton, Florida, pp. 321-347, 1997.

[15] Barlow, J.B., Rae, Jr. W.H., Pope, A. Low-speed wind tunnel testing. John W. \& Sons, INC, New York. 3rd edition, 1999.

[16] Jorgensen, F. E. How to measure turbulence with hot wire anemometers. Dantec Dynamic, Publication no.: 9004U6151. Denamark, pp. 40-44, 2002.

[17] Bruun, H. H. Hot-wire anemometry principles and signal analysis. Oxford Science Publications, pp234-239, 1995.

[18] Tummers, M. J., Flikweer, M. A., Hanjalic, K., Rodink, R. \& Moshfegh, B. Impiniging Jet cooling of a wall mounted cubes. Engineering Turbulence Modelling and Experiments 6, pp. 773-791, 2005 